\title{
Turbo Detection in RAyleigh flat FAding Channel With UnkNown STATISTICS
}

\author{
Mohamed Lassaad AMMARI ${ }^{1}$, Paul Fortier ${ }^{2}$ and Huu Tuê Huynh ${ }^{3}$ \\ ${ }^{1} 6$ 'Tel Research Unit, School of Communications of Tunis, $7^{\text {th }}$ November University at Carthage, \\ Tunis, Tunisia \\ mohamed.ammari@eniso.rnu.tn \\ 2,3 Department of Electrical and Computer Engineering, Laval University, Québec, Canada \\ 2 fortier@gel.ulaval.ca, ${ }^{3}$ huynh@gel.ulaval.ca
}

\begin{abstract}
The turbo detection of turbo coded symbols over correlated Rayleigh flat fading channels generated according to Jakes' model is considered in this paper. We propose a method to estimate the channel signal-to-noise ratio (SNR) and the maximum Doppler frequency. These statistics are required by the linear minimum mean squared error (LMMSE) channel estimator. To improve the system convergence, we redefine the channel reliability factor by taking into account the channel estimation error statistics. Simulation results for rate $1 / 3$ turbo code and two different normalized fading rates show that the use of the new reliability factor greatly improves the performance. The improvement is more substantial when channel statistics are unknown.
\end{abstract}

\section{KEYWORDS}

MAP-BCJR decoder, SNR, maximum Doppler frequency, turbo processing, channel reliability.

\section{INTRODUCTION}

Turbo codes can perform near the Shannon capacity limit in the AWGN channel [1]. They also give good performances for the Rayleigh flat fading channels with perfect knowledge of the Rayleigh process [2-6]. However in practice, the channel response is not available and has to be estimated. Therefore, in general, one estimates the channel gain and uses the estimated values to compute the channel reliability factor required by the maximum a posteriori probability MAP-BCJR algorithm. The design and the analysis of a receiver for turbo coded symbols detection in Rayleigh fading channels have been investigated by several researchers [2-5].

Recently, an iterative (turbo) detector has been proposed in [4-8] for joint channel estimation and turbo decoding. The turbo detector is a soft-in/soft-out (SISO) algorithm and the information exchanged between the channel estimator and the MAP decoder takes soft values. The channel estimation is the Wiener filter based on the minimum mean squared error (MMSE) criterion. Nevertheless, previous researches consider that the channel statistics are known.

This paper investigates a turbo detection technique of turbo coded symbols transmitted over correlated Rayleigh flat fading channel generated according to Jakes' model [9]. We assume that the channel SNR and the maximum Doppler frequency are unknown. We propose a procedure to estimate these parameters. This procedure uses the received signal statistics and a soft information fed back from the decoder. To improve the system convergence, we redefine the channel reliability factor. The proposed channel reliability expression takes into account the channel estimation error. We note that when the channel SNR is unknown, the Maximum-Likelihood (ML) channel estimator can be used. In fact, the ML estimator does not need the SNR. In this case we can omit the channel statistics estimation. However, for our turbo coded system, the SNR is necessary to compute the LLRs. For this reason, we propose to estimate the channel statistics and use the LMMSE fading gain estimator. Indeed, given perfect knowledge of the data sequence and the channel statistics, the LMMSE estimator is optimal in terms of minimum MSE. Of course, the performance of the LMMSE estimator depends on the channel statistics estimation accuracy.

DOI : $10.5121 /$ ijwmn.2010.2401 
Monte Carlo simulation results prove that the use of the redefined channel reliability improves the BER performance. We also demonstrate the efficiency of the proposed channel statistics estimation procedure.

\section{SYSTEM AND CHANNEL MODELS}

This paper uses the baseband system model shown in Fig. 1. The binary source sequence $\left\{d_{i}\right\}$ of length $L_{d}$ is encoded using a rate $r$ turbo code. Next, the encoded sequence is interleaved and known pilot symbols are periodically inserted. We note by $M_{p}$ the pilot spacing (number of data symbols between two consecutive pilot symbols). The data frame of length $N$ is BPSK modulated and transmitted over a correlated Rayleigh flat fading channel with additive white Gaussian noise (AWGN). Let us denote $x_{k}$ the transmitted signal, $T_{s}$ the symbol period and $g_{k}$ the multiplicative distortion of the flat fading channel. The received signal at time $k T_{s}$ is then

$$
y_{k}=g_{k} x_{k}+n_{k}, \quad 1 \leq k \leq N
$$

where $n_{k}$ is a zero-mean AWGN with variance $\sigma_{n}^{2}=N_{0} / 2$. The Rayleigh fading process is generated according to Jakes' model [9]. So, $g_{k}$ is a correlated complex circular Gaussian process with zero mean and variance $\sigma_{g}^{2}$. The fading autocorrelation function is determined by the maximum Doppler frequency $f_{M}$ as $[9]$

$$
\begin{aligned}
r_{g}(k) & =E\left\{g_{n} g_{n-k}^{*}\right\} \\
& =\sigma_{g}^{2} J_{0}\left(2 \pi f_{M} T_{s} k\right)=\sigma_{g}^{2} J_{0}\left(2 \pi f_{d} k\right)=\sigma_{g}^{2} \rho_{k}
\end{aligned}
$$

where $J_{0}($.$) is the zeroth-order Bessel function of the first kind, f_{d}$ is the maximum normalized Doppler frequency and ()* denotes complex conjugate. The real and imaginary parts of $g_{k}$ are supposed to be mutually uncorrelated. This very popular model for Rayleigh flat fading channels is often used in the literature [3-5]. It is noted that the transmitted signal is unit power and the average received SNR is given by

$$
\bar{\gamma}=\frac{\sigma_{g}^{2}}{\sigma_{n}^{2}}
$$

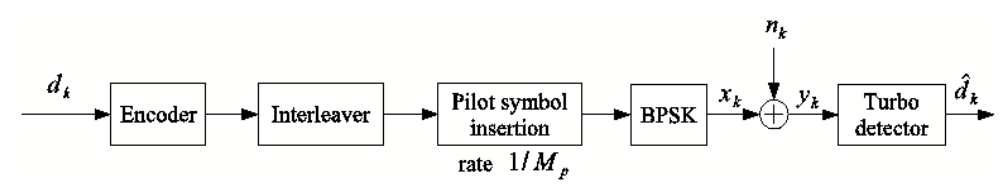

Figure 1: Transmission scheme.

\section{TURBO RECEPTION}

Classical receivers are formed by the concatenation of several disjoint modules (demodulator, decoder, equalizer ... ), each of which gives a hard decision as output. This approach has been shown to be suboptimal, because it uses only a small part of the available information [10]. For instance, the equalizer does not use the redundancy introduced by the decoder [10]. Moreover, hard decision at any module's output results in a loss of information. This suboptimality has motivated the idea of an iterative joint processing where soft information is exchanged between modules. This principle, called "turbo-detection", is inspired from the turbo decoding algorithm proposed in [1] and was proposed first in [10] for turbo equalization. Recently, turbo processing has been used for joint turbo decoding and Rayleigh flat fading channel estimation [5]. The Turbo detector in this context is based on the MAP decoder and the linear minimum mean-squared-error (LMMSE) channel estimator as illustrated in Figure (2). 


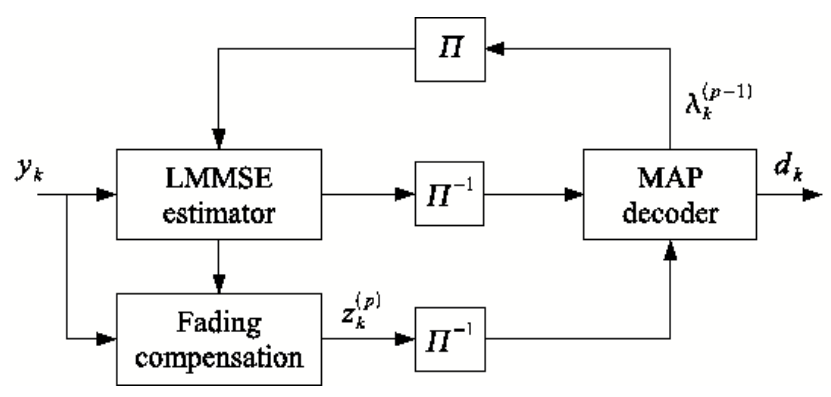

Figure 2: Turbo detector structure.

The well known BJCR-MAP decoding algorithm is used to compute the log likelihood ratio (LLR) of both information and coded symbols defined by

$$
\Lambda\left(c_{k}\right)=\frac{p\left(c_{k}=+1 \mid \boldsymbol{y}\right)}{p\left(c_{k}=-1 \mid \boldsymbol{y}\right)}
$$

where $\boldsymbol{y}=\left[y_{1}, y_{2}, \cdots, y_{N}\right]^{\mathrm{T}}$ is a column vector which represents the received sequence and the superscript $\mathrm{T}$ denotes the transpose operator. This algorithm needs the channel reliability factor which depends on $\boldsymbol{g}=\left[g_{1}, \cdots, g_{N}\right]^{\mathrm{T}}$. This Rayleigh channel realization is unknown and has to be estimated. The decoding and channel estimation are done using an iterative process. At any iteration, the channel estimator uses the decoder and the channel outputs and provides a LMMSE estimate of $\boldsymbol{g}$. The MAP decoder then uses these estimates to re-evaluate the LLR of each symbol. This iteration process repeats until the data decoding converges or until a processing delay limit is reached. It is noted that the information exchanged between modules is soft.

\section{Channel EStimation}

\subsection{LMMSE channel estimation}

The estimation of Rayleigh flat fading channels has been widely investigated in the literature [5,11]. In this paper, we use the well known pilot symbol assisted modulation (PSAM) technique [5, 11]. For this method, known pilot symbols are periodically inserted into the data sequence. For the proposed channel estimation technique, pilot symbols are used to initialize the channel fading estimator at the first iteration. Under a given SNR and a known maximum Doppler frequency, the optimal LMMSE channel gain estimator is the Wiener filter [11]. Authors in [5] have compared performances of the Wiener filter and the moving average filter. It has been shown that the Wiener filter outperforms the moving average one specially for fast fade rates.

At any iteration $(p>1)$, the channel fading can be extracted by dividing the received signal by the estimation of the transmitted signal

$$
r_{k}^{(p)}=y_{k} / \hat{x}_{k}^{(p-1)}
$$

where $\hat{x}_{k}^{(p-1)}$ is the expectation of the transmitted symbol $x_{k}$ at iteration $(p-1)$. This soft decision is given by [5]

$$
\begin{aligned}
\hat{x}_{k}^{(p-1)} & =\mathrm{E}\left\{x_{k} \mid \Lambda^{(p-1)}\left(x_{k}\right)\right\} \\
& =\tanh \left(\frac{\Lambda^{(p-1)}\left(x_{k}\right)}{2}\right)
\end{aligned}
$$

where $\Lambda^{(p-1)}\left(x_{k}\right)$ is the LLR of symbol $x_{k}$ delivered by the decoder at iteration $(p-1)$.

To estimate channel coefficients, scaled samples $r_{k}^{(p)}$ are used in order to minimize the mean square error (MSE)

$$
\mathrm{E}\left\{\left|\hat{g}_{k}^{(p)}-g_{k}\right|^{2} \mid \hat{x}_{1}^{(p-1)}, \cdots, \hat{x}_{N}^{(p-1)}\right\}
$$


where $\hat{g}_{k}^{(p)}$ denotes the estimate of $g_{k}$ at iteration $(p)$.

The mean square solution is given by

$$
\hat{g}_{k}^{(p)}=\left[\boldsymbol{W}^{(p)}\right]^{\mathrm{T}} \boldsymbol{r}_{k}^{(p)}
$$

where $\boldsymbol{r}_{k}^{(p)}=\left[r_{k-\lfloor(M-1) / 2\rfloor}^{(p)}, \cdots, r_{k+\lfloor M / 2\rfloor}^{(p)}\right]^{\mathrm{T}}$ and $\boldsymbol{W}^{(p)}=\left[w_{1}^{(p)}, \cdots, w_{M}^{(p)}\right]^{\mathrm{T}}$ is the set of $M$ filter coefficients obtained by solving the Wiener-Hopf equations [12]. The optimum Wiener filter is given by [12]

$$
\left[\boldsymbol{W}^{(p)}\right]^{\mathrm{H}}=\boldsymbol{R}_{g \boldsymbol{r}}^{(p)}\left[\boldsymbol{R}_{\boldsymbol{r} \boldsymbol{r}}^{(p)}\right]^{-1}
$$

where the superscript $\mathrm{H}$ refers to the Hermitian operator, $\boldsymbol{R}_{g \boldsymbol{r}}^{(p)}=\mathrm{E}\left\{g_{k}\left[\boldsymbol{r}_{k}^{(p)}\right]^{H}\right\}$ a row vector and $\boldsymbol{R}_{\boldsymbol{r} \boldsymbol{r}}^{(p)}=\mathrm{E}\left\{\boldsymbol{r}_{k}^{(p)}\left[\boldsymbol{r}_{k}^{(p)}\right]^{H}\right\}$ an $M \times M$ Toeplitz matrix,

Now we have to evaluate the correlation matrices $\boldsymbol{R}_{g \boldsymbol{r}}^{(p)}$ and $\boldsymbol{R}_{\boldsymbol{r} \boldsymbol{r}}^{(p)}$. Let us suppose that the symbols $\left\{x_{k}\right\}$ are independent. So, for all $i \in\left\{-\left\lfloor\frac{M-1}{2}\right\rfloor, \cdots,\left\lfloor\frac{M}{2}\right\rfloor\right\}$ and $j \in\left\{-\left\lfloor\frac{M-1}{2}\right\rfloor, \cdots,\left\lfloor\frac{M}{2}\right\rfloor\right\}$ we can write

$$
\boldsymbol{R}_{g \boldsymbol{r}}^{(p)}(i)=\mathrm{E}\left\{g_{k}\left[r_{k+i}^{(p)}\right]^{*}\right\}=\mathrm{E}\left\{g_{k} g_{k+i}^{*}\right\} \mathrm{E}\left\{\frac{x_{k+i}}{\hat{x}_{k+i}^{(p-1)}}\right\}
$$

and

$$
\begin{aligned}
\boldsymbol{R}_{\boldsymbol{r} \boldsymbol{r}}^{(p)}(i, j)= & \boldsymbol{R}_{\boldsymbol{r r}}^{(p)}(|i-j|) \\
= & \mathrm{E}\left\{r_{n+i}^{(p)}\left[r_{n+j}^{(p)}\right]^{*}\right\} \\
= & \mathrm{E}\left\{g_{n+i} g_{n+j}^{*}\right\} \mathrm{E}\left\{\frac{x_{n+i}}{\hat{x}_{n+i}^{(p-1)}}\right\} \mathrm{E}\left\{\frac{x_{n+j}}{\hat{x}_{n+j}^{(p-1)}}\right\} \\
& +\mathrm{E}\left\{n_{b+i} n_{n+j}\right\} \mathrm{E}\left\{\frac{1}{\hat{x}_{n+i}^{(p-1)} \hat{x}_{n+j}^{(p-1)}}\right\}
\end{aligned}
$$

where $\boldsymbol{R}_{g \boldsymbol{r}}^{(p)}(i)$ denotes element $i$ of vector $\boldsymbol{R}_{g \boldsymbol{r}}^{(p)}$ and $\boldsymbol{R}_{\boldsymbol{r} \boldsymbol{r}}^{(p)}(i, j)$ is the element $(i, j)$ of $\boldsymbol{R}_{\boldsymbol{r} \boldsymbol{r}}^{(p)}$. It has been proven that $\mathrm{E}\left\{x_{j} / \hat{x}_{j}^{(p-1)}\right\}$ can be approximated by [13]

$$
\begin{aligned}
\mathrm{E}\left\{x_{j} \hat{x}_{j}^{(p-1)}\right\} & \approx \frac{1}{N} \sum_{k=1}^{N}\left|\hat{x}_{k}^{(p-1)}\right|^{-1} \\
& =\frac{1}{N} \sum_{k=1}^{N}\left|\tanh \left(\frac{\Lambda\left(x_{k}\right)}{2}\right)\right|^{-1}
\end{aligned}
$$

In the same way, $\mathrm{E}\left\{1 / \hat{x}_{n+j}^{(p-1)} \hat{x}_{n+j}^{(p-1)}\right\}$ can be approximated by

$$
\mathrm{E}\left\{\frac{1}{\hat{x}_{n+j}^{(p-1)} \hat{x}_{n+j}^{(p-1)}}\right\} \approx \frac{1}{N} \sum_{k=1}^{N}\left|\tanh \left(\frac{\Lambda\left(x_{k}\right)}{2}\right)\right|^{-2}
$$

For the first iteration, only pilot symbols are known at the receiver. In this situation, the fading is extracted only at the pilot symbol times. The estimation filter size is then reduced from $M$ to $M / M_{p}$. The fading at the $k$ th data symbol time is estimated using the $M / M_{p}$ nearest pilot symbols.

\subsection{Channel statistics estimation}

The implementation of the Wiener filter requires the knowledge of the channel SNR $\left(\bar{\gamma}=\sigma_{g}^{2} / \sigma_{n}^{2}\right)$ and the maximum normalized Doppler frequency $f_{d}$ which are unknown in practice. So, to evaluate $\boldsymbol{R}_{g \boldsymbol{r}}^{(p)}$ and $\boldsymbol{R}_{\boldsymbol{r} \boldsymbol{r}}^{(p)}$, we have to estimate these channel statistics. 
Let us define the function $\phi(k)$ by

$$
\phi(k)=\frac{\boldsymbol{R}_{\boldsymbol{y} \boldsymbol{y}}(k)}{\boldsymbol{R}_{\boldsymbol{y} \boldsymbol{y}}(0)}
$$

which can be expressed as

$$
\begin{aligned}
\phi(k) & =\frac{\sigma_{g}^{2} \rho_{k}}{\sigma_{g}^{2}+\sigma_{n}^{2}}=\frac{\bar{\gamma} \rho_{k}}{\bar{\gamma}+1} \\
& =\frac{\bar{\gamma}}{\bar{\gamma}+1} J_{0}\left(2 \pi f_{d} k\right)
\end{aligned}
$$

The autocorrelation function of the scaled received sequence can be estimated as

$$
\hat{\boldsymbol{R}}_{\boldsymbol{y} \boldsymbol{y}}(k)=\frac{1}{N-k} \sum_{i=1}^{N-k} y_{i}^{(p-1)}\left[y_{i+k}^{(p-1)}\right]^{*}
$$

Using (16) and (15), we can estimate $\phi(k)$ by

$$
\hat{\phi}(k) \approx \frac{\hat{\boldsymbol{R}}_{\boldsymbol{y} \boldsymbol{y}}(k)}{\hat{\boldsymbol{R}}_{\boldsymbol{y} \boldsymbol{y}}(0)}
$$

Let us denote by $\theta_{1}=\bar{\gamma}$ and $\theta_{2}=f_{d}$ the two parameters to be estimated. Our statistics estimation procedure consists of finding the optimal vector $\boldsymbol{\theta}_{\mathrm{opt}}=\left[\theta_{1}, \theta_{2}\right]^{\mathrm{T}}$ that minimizes the total mean square error given by

$$
J=\mathrm{E}\{\mathcal{F}(\boldsymbol{\theta})\}
$$

where $\mathcal{F}(\boldsymbol{\theta})$ is defined as

$$
\mathcal{F}(\boldsymbol{\theta})=\sum_{k=1}^{Q}\left[\frac{\theta_{1}}{\theta_{1}+1} J_{0}\left(2 \pi \theta_{2} k\right)-\hat{\phi}(k)\right]^{2}
$$

where $Q$ is an integer in $\{2,3, \cdots, N-1\}$.

Using the least mean square (LMS) approach combined with the classical Newton's method, we can construct a recursive algorithm which would give, once it converges, the optimal solution $\boldsymbol{\theta}_{\mathrm{opt}}$. Let $\boldsymbol{\theta}^{(l)}$ the value of $\boldsymbol{\theta}$ at Newton's algorithm iteration $(l)$. Using the Taylor series expansion of $\mathcal{F}$ about $\boldsymbol{\theta}^{(l)}$ and neglecting terms of order three and higher, we have [14]

$$
\begin{aligned}
\mathcal{F}(\boldsymbol{\theta}) & =\mathcal{F}\left(\boldsymbol{\theta}^{(l)}\right)+\left(\boldsymbol{\theta}-\boldsymbol{\theta}^{(l)}\right)^{\prime} \nabla \mathcal{F}\left(\boldsymbol{\theta}^{(l)}\right) \\
& +\frac{1}{2}\left(\boldsymbol{\theta}-\boldsymbol{\theta}^{(l)}\right)^{\prime} \boldsymbol{F}\left(\boldsymbol{\theta}^{(l)}\right)\left(\boldsymbol{\theta}-\boldsymbol{\theta}^{(l)}\right)
\end{aligned}
$$

where $\nabla \mathcal{F}($.$) and \boldsymbol{F}$ are respectively the gradient and the Hessian matrix of $\mathcal{F}($.$) at \boldsymbol{\theta}^{(l)}$ given by

$$
\begin{aligned}
\nabla \mathcal{F} & =\left[\begin{array}{ll}
\frac{\partial \mathcal{F}}{\partial \theta_{1}}, & \frac{\partial \mathcal{F}}{\partial \theta_{2}}
\end{array}\right]^{\mathrm{T}} \\
\boldsymbol{F} & =\left(\begin{array}{ll}
\frac{\partial^{2} \mathcal{F}}{\partial \theta_{1}^{2}} & \frac{\partial^{2} \mathcal{F}}{\partial \theta_{1} \theta_{2}} \\
\frac{\partial^{2} \mathcal{F}}{\partial \theta_{2} \theta_{1}} & \frac{\partial^{2} \mathcal{F}}{\partial \theta_{2}^{2}}
\end{array}\right)
\end{aligned}
$$

So, the $l$ th iteration of Newton's method can be written as [14]

$$
\boldsymbol{\theta}^{(l+1)}=\boldsymbol{\theta}^{(l)}-\boldsymbol{F}\left(\boldsymbol{\theta}^{(l)}\right)^{-1} \nabla \mathcal{F}\left(\boldsymbol{\theta}^{(l)}\right)
$$

We note that, in practice, the maximum Doppler shift $f_{d}$ is much smaller than the symbol rate $F_{s}=1 / T_{s}$, so that $f_{d} T_{s} \ll 1$. This practical condition ensures the one-to-one mapping between $J_{0}\left(4 \pi f_{d} T_{s}\right)$ and $f_{d} T_{s}$. In fact, $J_{0}\left(4 \pi f_{d} T_{s}\right)$ is a monotonically decreasing function for $f_{d} T_{s}$ in $[0,0.1913]$, where 0.1913 corresponds to the first zero of Bessel function. 


\section{$5 \quad$ Modification of turbo decoding}

Once $\hat{\boldsymbol{g}}^{(p)}=\left[g_{1}^{(p)}, \cdots, g_{N}^{(p)}\right]^{\mathrm{T}}$ is evaluated, it is interleaved and passed to the decoder. The channel fading is then compensated by the fading estimate. Thus, at iteration $(p)$, the decoder evaluates the LLR for each transmitted symbol $x_{k}$ using the decision variable

$$
z_{k}^{(p)}=y_{k} / \hat{g}_{k}^{(p)}
$$

This decision variable depends on the channel estimate given by (8) which can be expressed as

$$
g_{k}=\hat{g}_{k}^{(p)}+e_{k}^{(p)}
$$

where $e_{k}^{(p)}$ denotes the channel estimation error at iteration $(p)$. We can easily prove that $e_{k}^{(p)}$ is a complex Gaussian random variable with $\mathrm{E}\left\{e_{k}^{(p)}\right\}=0$, which means that the the channel estimator is unbiased. For the ideal case where $\hat{x}_{k}^{(p-1)}=x_{k}$, the channel estimation error variance is given by $[12]$

$$
\sigma_{e}^{2}=\mathrm{E}\left\{\left|\hat{g}_{k}^{(p)}-g_{k}\right|^{2}\right\}=\sigma_{g}^{2}-\boldsymbol{R}_{g r}^{(p)}\left[\boldsymbol{R}_{r r}^{(p)}\right]^{-1}\left[\boldsymbol{R}_{g r}^{(p)}\right]^{\prime}
$$

The only parameter used by the MAP-BCJR decoding algorithm that depends on the decision variable statistics is called the channel reliability factor and is often denoted by $L_{c}$. It has been shown in [3] and [13] that turbo decoder performances can be improved by considering the channel estimation error when evaluating the channel reliability factor. The authors in $[5,13]$ do not discuss the evaluation of this metric and $L_{c}=2 / \sigma_{n}^{2}$ was used although the channel estimation is not perfect. This $L_{c}$ expression becomes inaccurate if the channel estimation error variance increases $[3,13]$. In the following, we present a new channel reliability factor that takes into account the estimation error variance.

The decision variable can be written as

$$
\begin{aligned}
z_{k}^{(p)} & =\frac{y_{k}}{\hat{g}_{k}^{(p)}}=\frac{x_{k}\left(\hat{g}_{k}^{(p)}+e_{k}^{(p)}\right)+n_{k}}{\hat{g}_{k}^{(p)}} \\
& =x_{k}+\frac{x_{k} e_{k}^{(p)}+n_{k}}{\hat{g}_{k}^{(p)}}
\end{aligned}
$$

So, the conditional probability density function of the decision variable given $\hat{g}_{k}^{(p)}$ and $x_{k}=a_{i}\left(a_{i}=\right.$ $\pm 1)$ is

$$
f\left(z_{k}^{(p)} \mid \hat{g}_{k}^{(p)}, x_{k}=a_{i}\right)=\frac{\left|\hat{g}_{k}^{(p)}\right|^{2}}{2 \pi \sigma_{u}^{2}} \exp \left(\frac{-\left|z_{k}-a_{i}\right|^{2}}{2 \sigma_{u}^{2} /\left|\hat{g}_{k}^{(p)}\right|^{2}}\right)
$$

where $\sigma_{u}^{2}$ can be approximated by $\sigma_{u}^{2} \approx \sigma_{n}^{2}+\sigma_{e}^{2}$. It is noted that, for this approximation, we assume that the AWGN $n_{k}$ and the estimation error $e_{k}^{(p)}$ are independent.

Given equation (28), we can write

$$
\ln \left\{\frac{f\left(z_{k}^{(p)} \mid \hat{g}_{k}^{(p)}, x_{k}=+1\right)}{f\left(z_{k}^{(p)} \mid \hat{g}_{k}^{(p)}, x_{k}=-1\right)}\right\}=\frac{2\left|\hat{g}_{k}^{(p)}\right|^{2}}{\sigma_{u}^{2}} \operatorname{Re}\left\{z_{k}^{(p)}\right\}
$$

where $\operatorname{Re}\left\{z_{k}^{(p)}\right\}$ is the real part of $z_{k}^{(p)}$.

Then, the channel reliability factor is

$$
L_{c}(k)=\frac{2\left|\hat{g}_{k}^{(p)}\right|^{2}}{\sigma_{u}^{2}}
$$




\section{Simulation ANALYSIS}

\subsection{Simulated system}

The performance of the proposed receiver has been evaluated by Monte Carlo simulations. We have used a rate $1 / 3$ turbo code of constraint length $K=3$ with a generator matrix $(1,5 / 7)$ in octal form. Two normalized fading rates $f_{d} \in\{.05, .002\}$ have been considered and the frame size was fixed to $N=1024$. For the slower fade rate $\left(f_{d}=0.002\right)$, a pilot symbol spacing of $M_{p}=20$ was used. For the faster fade rate $\left(f_{d}=0.5\right)$, the pilot symbol spacing was fixed at $M_{p}=10$. The Wiener filter in equation (8) was of length $M=45$. We have simulated the following five detection scenarios:

- (S1) : CSI (channel state information) case with perfect channel knowledge which serves as a benchmark;

- (S2) : unknown channel coefficients and known channel statistics, and the reliability factor is given by $(30)$;

- (S3) : unknown channel coefficients and known channel statistics, and the reliability factor is $L_{c}=\frac{4\left|\hat{g}_{k}\right|}{N_{0}}$;

- (S4) : unknown channel coefficients and statistics, and the reliability factor is given by (30);

- (S5) : unknown channel coefficients and statistics, and the reliability factor is $L_{c}=\frac{4\left|\hat{g}_{k}\right|}{N_{0}}$.

\subsection{Influence of channel reliability}

Figures 3 and 4 give the BER performance of the different simulated scenarios after six iterations of turbo decoding. It is shown that for all simulated cases, the use of the redefined channel reliability factor improves the BER performances. In fact, the performances achieved by scenarios (S2) and (S4) are respectively better than those given by (S3) and (S5). For systems with known channel statistics, the gain between (S2) and (S3) is about $0.3 \mathrm{~dB}$ for a BER of $10^{-4}$ and $f_{d}=0.002$. This gain is more important if the channel statistics are unknown. Indeed the discrepancy between (S4) and (S5) is about $0.9 \mathrm{~dB}$ for a BER of $10^{-4}$ and $f_{d}=0.002$.

We notice that the performances are better when the normalized fading rate $f_{m}$ increases. In fact, as the normalized fading rate decreases, the average burst length increases since the correlation between the transmitted signals increases.

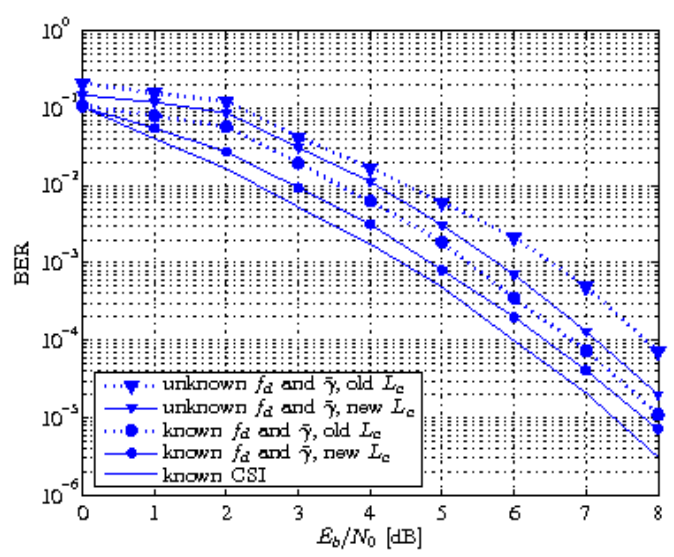

Figure 3: BER performance over flat fading channel with normalized fading rate $f_{d}=0.002$. 


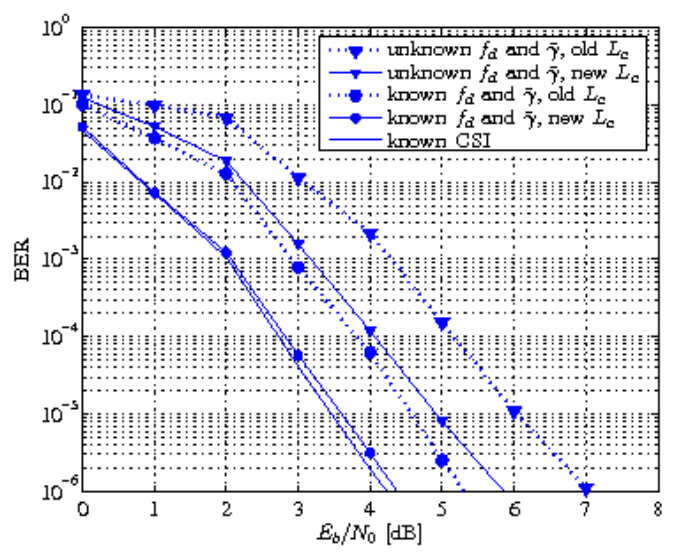

Figure 4: BER performance over flat fading channel with normalized fading rate $f_{d}=0.05$.

\subsection{Channel estimation}

As we can see in Figs. 3 and 4, the channel statistics estimation method presented in section 4.2 can help us to decode the transmitted sequence. Even though the channel statistics are unknown, we were able to estimate channel coefficients. The BER performances, obtained by combining the channel statistics estimation method and using the redefined channel reliability, are close to that of the CSI scenario.

Figures 5and 6 show the mean square error (MSE) of channel estimation respectively for $f_{m}=0.002$ and $f_{m}=0.05$. It is obvious that the MSE is less important when channel statistics are known. We note that unlike with the BER, the MSE performances are better when $f_{m}$ decreases.

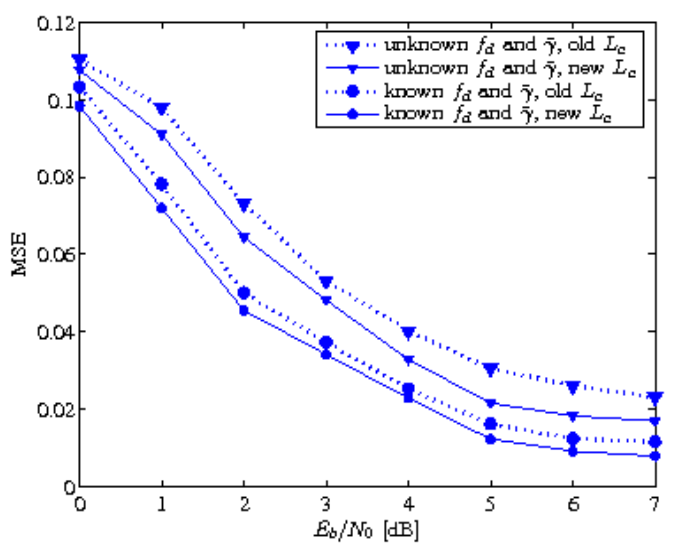

Figure 5: Mean square error (MSE) for $f_{d}=0.002$.

Figure 7 illustrates the mean square error of channel estimation after each iteration for $f_{d}=0.002$. We notice that there is a very important improvement in the MSE from the first iteration to the fourth one. However, after the fourth iteration, the improvement in MSE estimation is not noticeable for large values of $E_{b} / N_{0}$. So, it is possible to shut down the channel estimation after the fourth iteration.

To analyze the maximum Doppler frequency estimation accuracy, we define the normalized mean square error $N M S E_{f}$ as

$$
N M S E_{f}=\frac{\mathrm{E}\left\{\left|\hat{f}_{m}-f_{d}\right|^{2}\right\}}{f_{d}^{2}}
$$




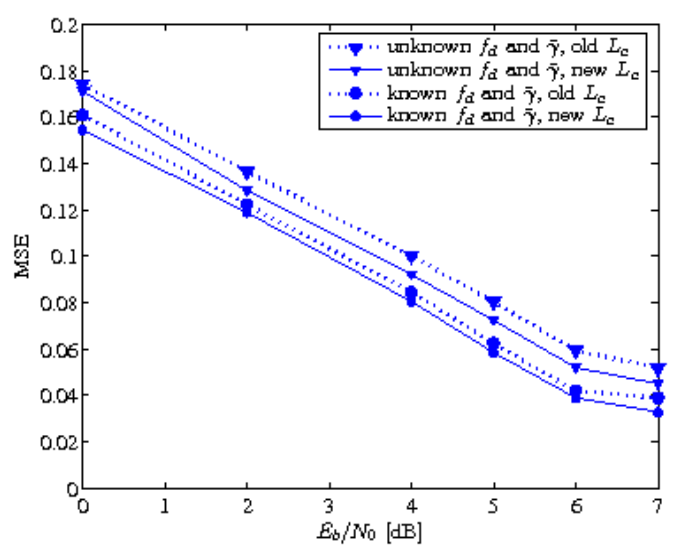

Figure 6: Mean square error (MSE) for $f_{d}=0.05$.

In Fig. 8, we have plotted $N M S E_{f}$ as a function of parameter $Q$ of Equation (19). Curves of Fig. 6 are obtained for $E_{b} / N_{0}=7 \mathrm{~dB}$ and $f_{d} \in\{0.002,0.05\}$. We note that $N M S E_{f}$ is better when the normalized fading rate increases. The improvement in $N M S E_{f}$ for $Q>5$ is insignificant.

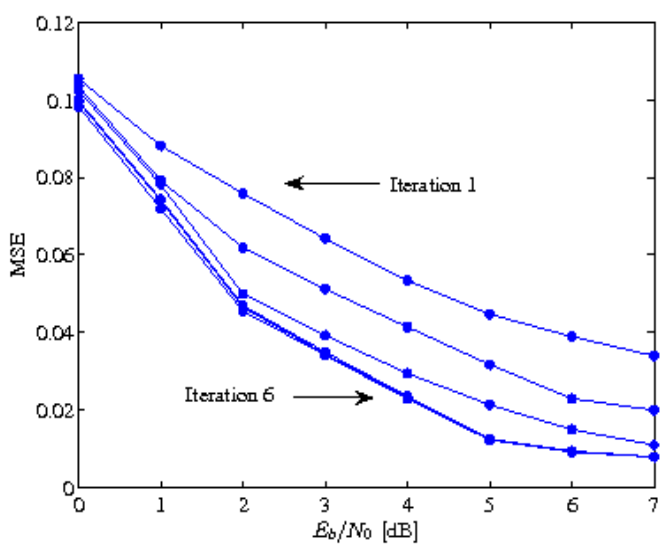

Figure 7: Mean square error (MSE) through iteration when $f_{d}=0.002$.

\section{CONCLUSION}

This paper focuses on turbo detection in Rayleigh flat fading channels with unknown statistics and coefficients. We have presented a turbo detector which combines MAP decoding and channel estimation. The channel coefficients estimator is based on the MMSE criterion. The MAP-BCJR algorithm is used for turbo decoding. The global receiver is an iterative SISO algorithm where different modules exchange soft information. The advantage of this procedure is that the channel estimator uses the redundancy information introduced by the channel encoder.

We have considered that channel SNR and the maximum Doppler frequency are unknown. An estimation method to evaluate these statistics has been presented. The proposed method uses the channel output signal and a soft information fed back from the decoder. Computer simulations have demonstrated the efficiency of the proposed channel statistics estimation procedure. It has been shown that through iterations, there is an improvement in the channel estimation accuracy. Since channel estimation is not perfect, we have suggested to redefine the channel reliability factor. The expression used for this metric takes into consideration the variance of the channel estimation 


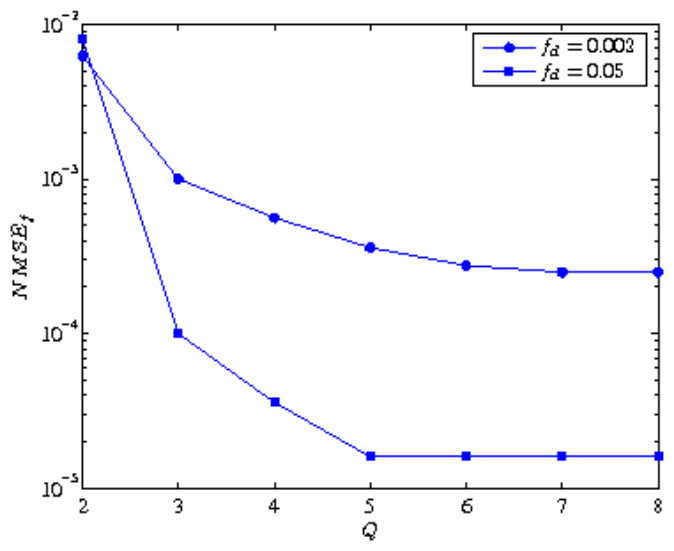

Figure 8: Normalized mean square error $N M S E_{f}$ vs. factor $Q$ of Equation (19) for $f_{d}=0.002$ and $f_{d}=0.05$.

error. When channel statistics are known, the error variance can be expressed in an analytical form. However, when these statistics are unknown, the channel reliability can be approximated, at any iteration, by using the channel output and the channel coefficients estimate evaluated at the previous iteration.

\section{References}

[1] C. Berrou, A. Glavieux, and P. Thitimajshima, "Near Shannon limit error correcting coding and decoding: Turbo-codes," in Proc., IEEE Int. Conf. on Commun., 1993, pp. 1064-1070.

[2] E. K. Hall and S. G. Wilson, "Design and Analysis of Turbo Codes on Rayleigh Fading Channels," IEEE J. Sel. Areas Commun., vol. 16, no. 2, pp. 160-174, Feb. 1998.

[3] P. Frenger, "Turbo decoding on Rayleigh fading channels with noisy channel estimates," in Proc., IEEE Veh. Technol. Conf., 1999, pp. 884-888.

[4] C. Komninakis and R. D. Wessel, "Pilot-aided joint data and channel estimation in flat correlated fading," in Proc., IEEE Global Telecomm. Conf., 1999, pp. 2534-2539.

[5] M. C. Valenti and B. D. Woerner, "Iterative channel estimation and decoding of pilot symbol assisted turbo codes over flat-fading channels," IEEE J. Sel. Areas Commun., vol. 19, no. 9, pp. 1697-1705, 2001.

[6] S. Lars, M. Heinrich, and Z. Dan, "Systematic design of iterative ML receivers for flat fading channels," IEEE Trans. Commun., vol. 58, no. 7, pp. 1897-1901, 2010.

[7] X. Jaspar and L. Vandendorpe, "Joint source-channel codes based on irregular turbo codes and variable length codes," IEEE Trans. Commun., vol. 56, no. 11, pp. 1824-1835, 2008.

[8] J. K. Hyosung Kim Tugnait, "Turbo equalization for doubly-selective fading channels using nonlinear kalman filtering and basis expansion models," IEEE Trans. Wireless Commun., vol. 9, no. 6, pp. 2076-2087, 2010.

[9] W. C. Jakes, Ed., Microwave Mobile Communications, 2nd ed. Wiley-IEEE Press, 51994.

[10] C. Douillard, M. Jézéquel, C. Berrou, A. Picart, P. Didier, and A. Glavieux, "Iterative correction of intersymbol interference: Turbo equalization," European Transactions on Telecommunications, vol. 6, no. 5, pp. 507-511, sep./oct. 1995. 
International Journal of Wireless \& Mobile Networks (IJWMN) Vol.2, No.4, November 2010

[11] J. Cavers, "An analysis of pilot symbol assisted modulation for rayleigh fading channels," IEEE Trans. Veh. Technol., vol. 40, no. 4, pp. 686-696, Nov. 1991.

[12] H. V. Poor, An Introduction to Signal Detection and Estimation, 2nd ed. Springer, 31998.

[13] J. W. Kim and W. S. Yoon, "Turbo code with iterative channel estimator using soft-output of turbo decoder," Electronics Letters, vol. 36, no. 18, pp. 1560-1562, Aug. 2000.

[14] K. P. C. Edwin and H. Z. Stanislaw, An Introduction to Optimization. Wiley-Interscience, 1995. 